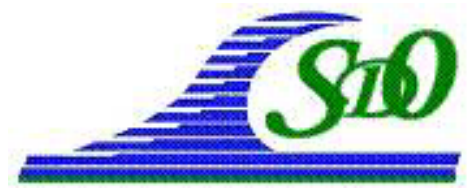

XI ${ }^{\text {èmes }}$ Journées Nationales Génie Côtier - Génie Civil

Les Sables d'Olonne, 22-25 juin 2010

DOI:10.5150/jngcgc.2010.072-P C Editions Paralia CFL

disponible en ligne - http://www.paralia.fr - available online

\title{
Vulnérabilité du territoire national aux risques littoraux
}

\section{Céline PERHERIN ${ }^{1}$, Amélie ROCHE ${ }^{1}$, Frédéric PONS ${ }^{2}$, Isabelle ROUX ${ }^{2}$, Guy DESIRE $^{3}$, Céline BOURA ${ }^{3}$}

1. Centre d'Etudes Techniques Maritimes et Fluviales, DELCE, DHSM, BP 5, 29280 Plouzané, France.

Celine.Perherin@developpement-durable.gouv.fr ;

Amelie.Roche@developpement-durable.gouv.fr

2. Centre d'Etudes Techniques de l'Equipement Méditerranée, DREC, SRILH, BP 37 000, 13791 Aix-En-Provence, France.

Frederic.Pons@developpement-durable.gouv.fr ;

Isabelle.Roux@developpement-durable.gouv.fr

3. Centre d'Etudes Techniques de l'Equipement de l'Ouest, DIE, Environnement,

M.A.N., Rue René Viviani, BP 46 223, 44262 Nantes Cedex 2, France.

Guy.Desire@developpement-durable.gouv.fr ;

Celine.Boura@developpement-durable.gouv.fr

\section{Résumé :}

La "Vulnérabilité du territoire national aux risques littoraux" est une étude pilotée par le Centre d'Études Techniques Maritimes et Fluviales (CETMEF) en 2008-2009 en collaboration avec les CETE Méditerranée et de l'Ouest. L'objectif était d'établir une représentation simple des actuelles zones vulnérables aux risques littoraux en France métropolitaine. Les principales études régionales et départementales ont fait l'objet d'une importante synthèse bibliographique ; la détermination de différents indicateurs, basés sur l'analyse de bases de données disponibles au niveau national (arrêtés de déclaration de l'état de catastrophe naturelle, niveaux marins extrêmes, BD Topo ${ }^{\circledR}$ IGN et enjeux), a permis de quantifier la vulnérabilité et de réaliser de nombreuses cartes de synthèse.

Mots-clés :

Vulnérabilité - Indicateurs - Risques littoraux - Aléas - Enjeux - Cartographie

\begin{abstract}
:
"French national territory vulnerability to coastal risks" is a study led in $2008-2009$ by CETMEF in close collaboration with two French public engineering centres (CETE Méditerranée and CETE de l'Ouest). The main objective was to map and estimate the expanse of the current French areas vulnerable to coastal hazards. Firstly the main regional-scaled studies were summarized to give general trends. Secondly different risk indicators, based on the analysis of public national databases, were determined, mapped and combined to quantify vulnerability.
\end{abstract}




\section{Introduction}

L'étude "Vulnérabilité du territoire national aux risques littoraux" a été lancée par le CETMEF en 2008, en partenariat avec les Centres d'Études Techniques de l'Équipement (CETE) de Méditerranée et de l'Ouest, afin de disposer d'une synthèse nationale des connaissances actuelles sur les risques littoraux (érosion des côtes basses meubles et des falaises, avancées dunaires, submersion marine) et d'évaluer les secteurs qui seront les plus susceptibles d'être impactés par l'élévation du niveau marin. On entend par "vulnérabilité du territoire" la conséquence physique des aléas côtiers sur un territoire exposé, c'est-à-dire, pour la submersion marine par exemple, l'inondation des terres par la mer et ses conséquences sur les enjeux. Cette étude (CETMEF - CETE Méditerranée - CETE Ouest, 2010) s'est déclinée en deux parties, une synthèse bibliographique d'une part, et la définition de méthodes permettant de quantifier la vulnérabilité et d'établir une représentation des principales zones vulnérables en France métropolitaine d'autre part, dont les principaux résultats sont présentés ci-dessous.

\section{Synthèse bibliographique}

\subsection{Les études sources}

La synthèse bibliographique a permis d'évaluer l'état des connaissances sur les risques littoraux et de faire une synthèse nationale de la vulnérabilité. Elle s'est appuyée sur les rapports d'études menées à l'échelle régionale ou départementale, principalement réalisées sous la maîtrise d'ouvrage des services déconcentrés du MEEDDM (DREAL, DDEA...), et traitant de la submersion marine et l'évolution du trait de côte, ou le cas échéant de la vulnérabilité du littoral liée au changement climatique. Les études universitaires couvrant un linéaire au moins départemental ont également été recensées. En tout 51 rapports d'étude ont été retenus, pour lesquels une "fiche-résumé" a été réalisée comprenant les métadonnées de l'étude (titre, année, auteur, commanditaire...), les thèmes abordés (aléas érosion et submersion marine, enjeux, vulnérabilité, gestion $\mathrm{du}$ littoral et méthodes de protection, impact du changement climatique) et la manière dont ils ont été traités, un résumé ainsi que la synthèse des principales informations extraites de l'étude concernant la vulnérabilité des territoires aux risques littoraux.

\subsection{La synthèse bibliographique}

Des synthèses régionales ont été réalisées à partir des études sources recensées et d'études plus locales, sans tenir compte des diverses analyses conduites dans le reste de l'étude. Elles se présentent par unités sédimentaires ou, du moins, secteurs homogènes.

Les informations disponibles le long des côtes françaises ne sont pas homogènes. Certains secteurs ont fait l'objet de multiples études alors que d'autres sont peu traités. Cinq régions se dégagent, le Nord-Pas-de-Calais, la Haute-Normandie, la BasseNormandie, la Bretagne et les Pays de La Loire, où l'ensemble des thèmes est traité. 
Une connaissance approfondie de la vulnérabilité aux risques littoraux sur certaines régions peut s'expliquer par une volonté politique forte ou la présence d'une université active sur ces thématiques. A l'inverse, ce qui peut apparaitre au premier abord comme un manque de connaissance peut simplement rendre compte de secteurs peu concernés par les risques littoraux. De plus, si de nombreuses études portent sur les aléas, peu portent sur les stratégies de gestion du littoral. L'impact du changement climatique a été étudié principalement en Haute-Normandie et dans le Nord-Pas-de-Calais.

\section{Indicateurs de vulnérabilité aux risques littoraux}

\subsection{Indicateur "Niveau de connaissance"}

L'indicateur "Niveau de connaissance" de la vulnérabilité aux risques littoraux a été déterminé à partir de la synthèse bibliographique et de l'évaluation du traitement des thèmes suivants : aléa érosion, aléa submersion marine, enjeux, vulnérabilité, méthodes de protection, impact du changement climatique. A partir de ces évaluations, l'indicateur "Niveau de connaissance" a été calculé par région et par thème, puis cartographié.

\subsection{Indicateur "Zones basses"}

La cartographie des zones basses, zones topographiques situées sous un niveau marin de référence, est un premier indicateur de la vulnérabilité du territoire aux submersions marines. La méthodologie de cartographie reprend celle de l'aléa submersion marine du guide méthodologique "Plans de Prévention des Risques Littoraux" (MATE \& METL, 1997) : on "superpose la cote du plan d'eau retenu à la topographie, pour cartographier les espaces continentaux situés à une altitude inférieure à la cote de référence". Les zones basses obtenues sont distinctes des zones inondables. Elles sont définies sans tenir compte des ouvrages de protection. De plus, seule une approche statique est adoptée (non prise en compte de la tenue du niveau de pleine mer et des franchissements).

Le traitement du Modèle Numérique de Terrain (MNT) de la BD Topo ${ }^{\circledR}$ de l'IGN a permis la création de polylignes d'iso-valeurs (valeurs semi-entières) et l'obtention de polygones pour le calcul des surfaces. Sur les côtes Atlantique-Manche-Mer du Nord, le niveau marin centennal est extrait de l'étude SHOM-CETMEF (SIMON, 2008). En cohérence avec la précision du MNT, seules les iso-valeurs de niveaux marins correspondant à des demi-valeurs entières (de $+2,5$ à $+8,5 \mathrm{~m} \mathrm{NGF}$ ) ont été retenues. Les limites des zones des différents niveaux marins ont été prolongées dans les terres perpendiculairement au trait de côte Histolitt (IGN-SHOM). En Méditerranée, la cote de référence $+1,5 \mathrm{~m}$ NGF a été choisie à partir de la bibliographie (DIREN LR, 2008; PONS \& GENTIL, 2007) et des possibilités offertes par le croisement du MNT et des niveaux marins. 28 classes ont ainsi été définies sur le littoral métropolitain. Les valeurs des niveaux de référence retenues pour cette étude ne remettent nullement en cause les valeurs potentiellement différentes retenues par les services. 
Compte-tenu de l'incertitude altimétrique du MNT, et d'étudier le possible impact d'élévation du niveau marin moyen lié au changement climatique (élévation à échéance 100 ans ici retenue : $+1 \mathrm{~m}$ ), trois types de zones ont été étudiées : les zones situées sous le niveau marin centennal, sous le niveau marin centennal moins 1 mètre et sous le niveau marin centennal plus 1 mètre, estimant ainsi les variations de surface en fonction des niveaux marins retenus. Les zones en eau, lacs, étangs, cours d'eau, ont été extraites des zones basses à partir de la couche des surfaces en eau de la BD Topo ${ }^{\circledR}$.

D'après les estimations effectuées, 590000 hectares sont situés sous les niveaux marins centennaux et 735500 ha sont situés sous les niveaux marins centennaux $+1 \mathrm{~m}$. Les régions dont les surfaces de zones basses sont les plus importantes sont les Pays de la Loire, le Poitou-Charentes et le Nord-Pas-de-Calais (voir figure 1). Les régions PACA, Aquitaine et Languedoc-Roussillon sont celles dont les surfaces de zones basses augmentent le plus avec une élévation du niveau marin.

La qualité des résultats obtenus dépend de la méthodologie et de la précision des données initiales, principalement du MNT dont l'incertitude en altimétrie est estimée à $+/-1 \mathrm{~m}$ (d'après valeurs fournies par l'IGN). Pour leur validation, les résultats des zones basses ont été comparés à ceux obtenus à partir d'un MNT de précision altimétrique décimétrique (MNT du Bassin de Thau), et aux zones obtenues par une approche géomorphologique (ESPOSITO et al., 2009). Les résultats sont comparables en ordre de grandeur des surfaces mais des différences ont été soulevées au niveau des zones les plus basses (comparaison avec le MNT du Bassin de Thau entre 0 et +1,5 m NGF).

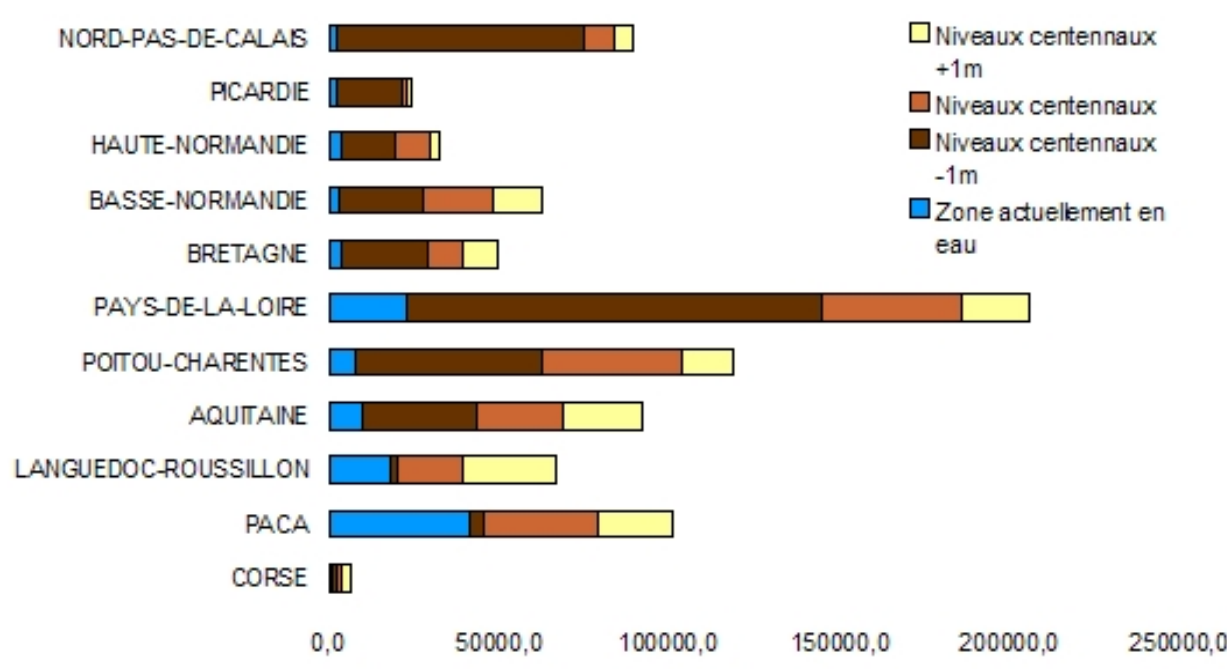

Figure 1. Surfaces des zones basses en eau (lacs, étangs...), situées sous les niveaux centennaux, sous les niveaux centennaux $-1 \mathrm{~m}$ et sous les niveaux centennaux $+1 \mathrm{~m}$.

\subsection{Arrêtés de reconnaissance de l'état de catastrophe naturelle liés à la mer}

La base de données GASPAR (Gestion Assistée des Procédures Administratives relatives aux Risques naturels et technologiques) recense par région les arrêtés intermi- 


\section{XI $I^{\text {èes }}$ Journées Nationales Génie Côtier - Génie Civil}

Les Sables d'Olonne, 22-25 juin 2010

nistériels pris lors d'événements exceptionnels reconnus comme catastrophes naturelles. Ces arrêtés constituent un indicateur d'exposition d'une commune à un risque, qui peut être littoral. L'exploitation de cette base de données a permis d'évaluer et de cartographier le nombre d'arrêtés par commune liés à des phénomènes marins. Un arrêté peut cependant être pris pour un seul comme un grand nombre de biens touchés.

La terminologie des risques inscrits dans la base a été traitée car les intitulés ne sont pas directement liés à l'action de la mer (intitulés type "tempête" ou "Inondations, coulées de boues, glissement et chocs mécaniques liés à l'action des vagues"). L'analyse a porté sur les arrêtés concernant les communes situées dans les zones basses définies ci-dessus. Un coefficient a été affecté à chacun des arrêtés retenus : "0" lorsque l'arrêté était jugé non lié à la mer, "1" lorsqu'il était jugé lié à la mer avec certitude (ou à l'action des vagues) ou si la commune était littorale ou riveraine d'estuaire et de delta (liste définie par le décret n 2004-311 du 29 mars 2004), "0,5" sinon.

Ont été exclus les risques non-associés à la mer (avalanches, séismes) et d'autres risques tels que les mouvements de terrain et effondrements de falaise par impossibilité de validation avec la base de données des mouvements de terrain gérée et développée par le BRGM avec le LCPC et le service Restauration des Terrains en Montagne (RTM).

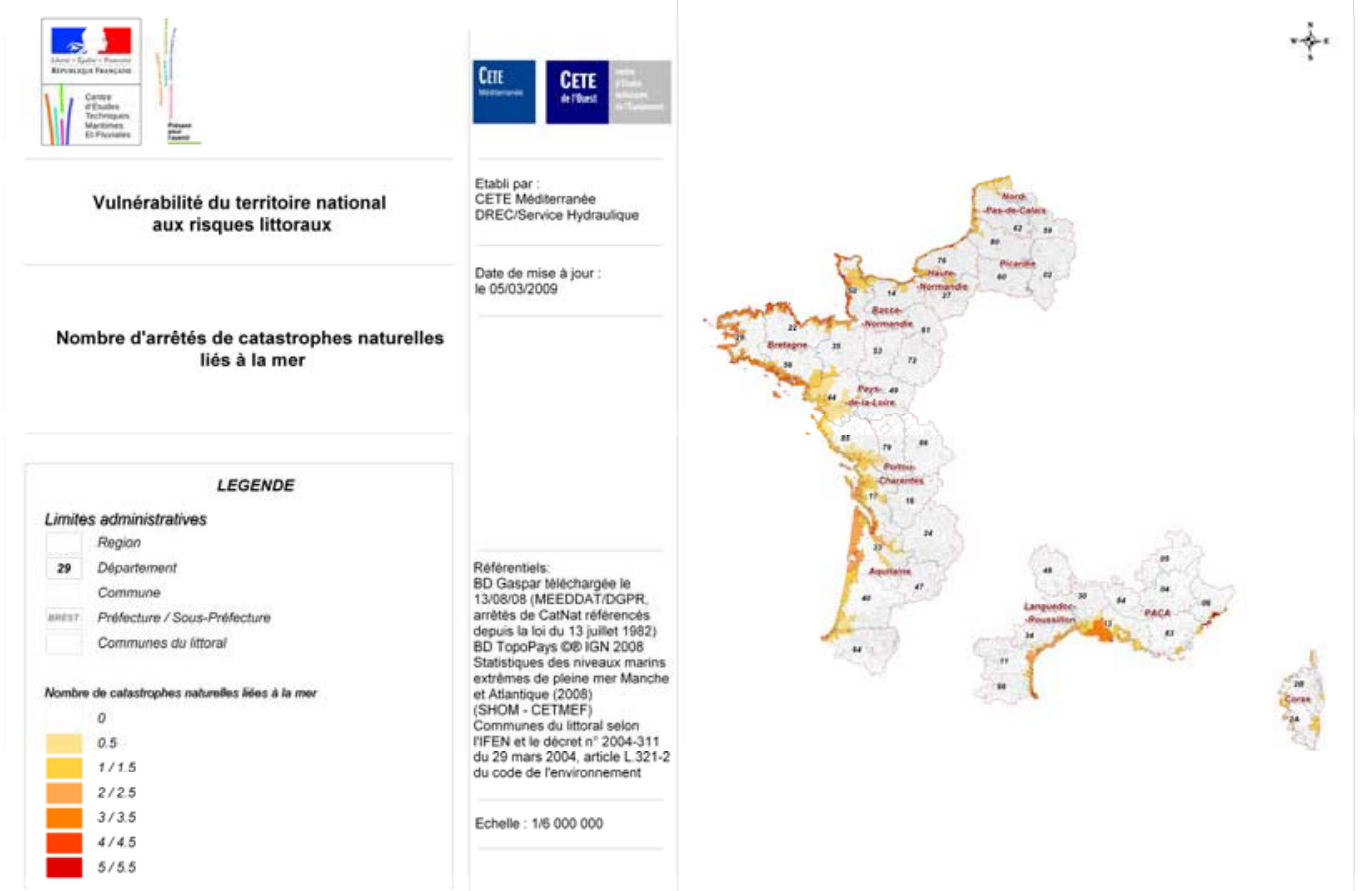

Figure 2. Carte des arrêtés de catastrophes naturelles liés à la mer.

Le nombre de catastrophes naturelles par commune a été déterminé en sommant des valeurs des coefficients attribués (voir figure 2). Les résultats ne sont pas valables au niveau local mais national, les intitulés étant attribués de façon assez libre. Les régions Bretagne et Basse-Normandie concentrent les communes ayant les plus grands nombres 
d'arrêtés. Les Alpes-Maritimes ressortent aussi, ainsi que les estuaires (Gironde et Seine). Les arrêtés couvrent l'ensemble des communes littorales des départements, à l'exception de la Corse et du Var.

Six ou sept événements majeurs ont eu lieu depuis 1982, date de la mise en place de la procédure. Le principal événement est la tempête de 1999 qui a touché les façades Atlantique et Manche. Les tempêtes de 1982 et 1987 ont touché respectivement la Méditerranée et l'Aquitaine, la Bretagne et la Basse-Normandie.

\subsection{Atlas de zones inondables et plans de prévention liés à la mer}

La présence de documents réglementaires ou d'informations sur un territoire peut révéler l'existence de risques reconnus. La base de données nationale GASPAR recense les documents d'information préventive ou à portée réglementaire, dont les Atlas de Zones Inondables (AZI) et les plans de préventions tels que les Plans de Prévention des Risques Littoraux (PPRL) ou des Risques Naturels (PPRn), les Plans d'Exposition aux Risques (PER) et les périmètres R111-3 (article qui permet d'interdire ou de limiter les constructions exposées à des nuisances graves).

La cartographie réalisée montre que ces documents sont plus présents sur les façades Atlantique et Manche. Cependant cette base n'est pas régulièrement renseignée et les informations issues de cet indicateur ne peuvent donc pas être utilisées directement.

\subsection{Enjeux situés dans les zones basses}

Un indicateur de vulnérabilité basé sur un certain nombre d'enjeux, pour lesquels des bases de données homogènes à l'échelle nationale étaient disponibles, a été défini. Ces enjeux ont été comptabilisés dans les zones basses, rendant ainsi compte d'un degré de vulnérabilité à la submersion marine. Il s'agit: des constructions (bâtiments administratifs, industriels, résidentiels...) et des infrastructures de transports (voies routières et ferroviaires) issues de la BD Topo Pays ${ }^{\circledR}$ de l'IGN, des établissements industriels à risques (classés SEVESO et centrales nucléaires) et des sites d'intérêt écologique issus du réseau Natura 2000. L'ensemble de ces enjeux a été déterminé pour chaque type de zones basses (sous les niveaux marins centennaux, $-1 \mathrm{~m}$ et $+1 \mathrm{~m}$ ) dans chaque région et département littoral métropolitain (voir tableau 1).

Les constructions et les infrastructures de transports concernent principalement les régions dont les surfaces de zones basses sont les plus élevées : le Nord-Pas-de-Calais, les Pays de la Loire, le Poitou-Charentes, le Languedoc-Roussillon et l'Aquitaine. Les établissements industriels à risques des communes littorales sont essentiellement situés dans les zones portuaires (Grands Ports Maritimes de Marseille, Le Havre, Rouen et Dunkerque). Les zonages de protections environnementales représentent près de 383000 ha et $64 \%$ des surfaces situées sous les niveaux marins centennaux, concernant essentiellement les Pays de la Loire, Poitou-Charentes et PACA. 
Tableau 1. Enjeux situés dans les zones basses sous les niveaux marins centennaux.

\begin{tabular}{llll}
\hline Régions & $\begin{array}{l}\text { Nombre de } \\
\text { bâtiments }\end{array}$ & $\begin{array}{l}\text { Linéaire } \\
\text { d'infrastructures } \\
\text { de transport (km) }\end{array}$ & $\begin{array}{l}\text { Surface de sites } \\
\text { écónét }\end{array}$ \\
\hline NORD PAS-DE-CALAIS & (ha) \\
PICARDIE & 65676 & 3757 & 1918 \\
HAUTE-NORMANDIE & 10149 & 545 & 5445 \\
BASSE-NORMANDIE & 9003 & 923 & 12410 \\
BRETAGNE & 15591 & 868 & 28923 \\
PAYS DE LA LOIRE & 22375 & 1611 & 15540 \\
POITOU-CHARENTES & 59658 & 3074 & 125901 \\
AQUITAINE & 28762 & 1783 & 64052 \\
LANGUEDOC-ROUSSILLON & 25916 & 1582 & 34367 \\
PROVENCE-ALPES-COTE D'AZUR & 16135 & 1905 & 27752 \\
CORSE & 10571 & 711 & 64973 \\
\hline FRANCE METROPOLITAINE & $\mathbf{2 6 5 1 5 6}$ & $\mathbf{1 6 8 0 4}$ & $\mathbf{3 8 2 6 4 0}$ \\
\hline
\end{tabular}

\subsection{Indicateur croisé}

Pour cartographier la vulnérabilité aux risques littoraux à l'échelle nationale, le croisement des différents indicateurs liés aux aléas et aux enjeux est un élément de réponse. Il a été choisi de croiser les arrêtés de catastrophes naturelles et les bâtiments situés dans les zones basses par commune, en considérant qu'une commune sera plus vulnérable si elle contient un grand nombre de bâtiments et qu'elle a déjà subi de nombreuses catastrophes naturelles d'origine marine. L'indicateur rend donc principalement compte de la vulnérabilité à la submersion marine. 6 classes ont été définies. Les régions les plus vulnérables sont celles dont les communes ont un niveau de vulnérabilité élevé (voir figure 3) qui sont surtout les communes littorales.

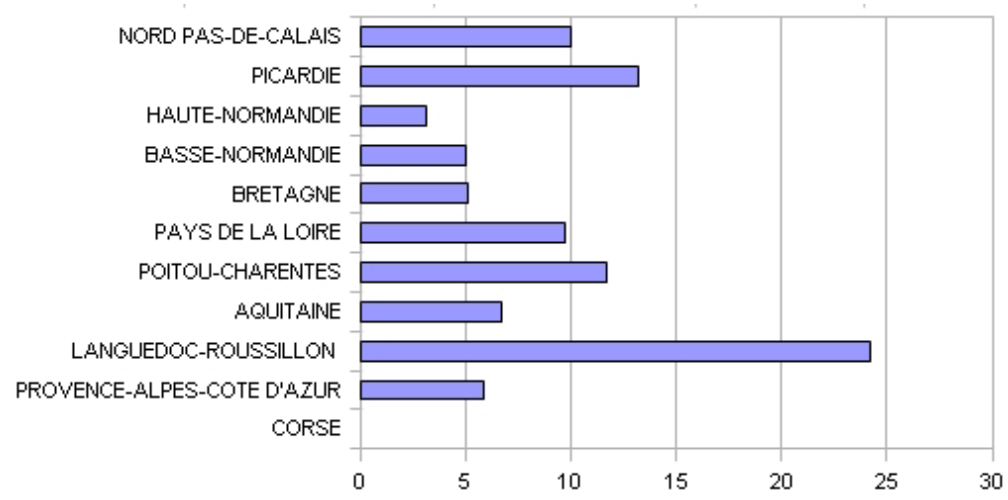

Figure 3. Indicateur croisé - Rapport par région du nombre de communes des classes 4 à 6 sur le nombre de communes situées en zones basses (sous le niveau centennal). 
Ce sont les régions du Languedoc-Roussillon, de la Picardie, du Poitou-Charentes, du Nord - Pas de Calais et des Pays de La Loire. A l'opposé, les régions Corse, HauteNormandie, Basse-Normandie et Bretagne sont moins vulnérables.

La cartographie a aussi été réalisée pour le bâti sous les niveaux marins centennaux $+1 \mathrm{~m}$ afin d'évaluer l'évolution de la vulnérabilité avec l'élévation du niveau moyen de la mer. La région où l'évolution est la plus forte est le Languedoc-Roussillon.

\section{Conclusion}

L'étude (CETMEF - CETE Méditerranée - CETE Ouest, 2010) a permis de faire un premier bilan des éléments disponibles pour faire une synthèse des risques littoraux à l'échelle métropolitaine. L'ensemble des principales études menées sur les risques littoraux en France ont été recensées et sont la base d'une synthèse bibliographique réalisée par région. Les bases de données présentant des informations homogènes à l'échelle nationale ont également été exploitées pour produire des indicateurs, données quantifiables ayant permis la réalisation de cartes à l'échelle nationale. Il est apparu qu'un indicateur seul ne donnait qu'une image partielle de la vulnérabilité. Un croisement des indicateurs a donc été proposé pour cartographier ainsi la vulnérabilité aux risques littoraux en France métropolitaine. L'ensemble des pistes évoquées au cours de l'étude n'a cependant pas pu être exploré et l'étude se poursuivra en 2010, en particulier avec l'extension aux DOM et aux COM.

\section{Références bibliographiques}

CETMEF - CETE Méditerranée - CETE Ouest. (2010). Vulnérabilité du territoire National aux risques littoraux. Rapport CETMEF/DELCE.163 p.

DIREN -Direction Régionale de l'Environnement- Languedoc-Roussillon (2008). Guide d'élaboration des PPR submersion marine en Languedoc- Roussillon. DIREN LR Préfecture de Région, $12 \mathrm{p}$.

ESPOSITO C., DELGADO J-L., PONS F. (2009). Atlas numérique des zones inondables par submersion marine. Littoral sableux du Languedoc-Roussillon. Élaboration d'un Système d'Information Géographique. CETE Méditerranée, DRE Direction Régionale de l'Equipement- Languedoc-Roussillon, 71 p.

MATE, METL -Ministère de l'Aménagement du Territoire et de l'Environnement, Ministère de l'Equipement, des Transports et du Logement- (1997). Plan de prévention des risques littoraux (PPR). Guide méthodologique. La documentation française, Paris, $54 \mathrm{p}$.

PONS F., LE GENTIL J. (2007). Élaboration d'un Plan de Prévention de Risques Submersions marines en Camargue - État des lieux des données existantes. CETE Méditerranée, DDE 13, 81 p.

SIMON B. (2008). Statistiques des niveaux marins extrêmes de pleine mer Manche et Atlantique. SHOM-CETMEF, Cédérom. 